\title{
Neutron Stars Phenomenology with Scalar-tensor Inflationary Attractors
}

\author{
S.D. Odintsov, ${ }^{1,2}$ V.K. Oikonomou, ${ }^{3,4}$ \\ 1) ICREA, Passeig Luis Companys, 23, 08010 Barcelona, Spain \\ 2) Institute of Space Sciences (IEEC-CSIC) C. Can Magrans s/n, 08193 Barcelona, Spain \\ 3) Department of Physics, Aristotle University of Thessaloniki, Thessaloniki 54124, Greece \\ 4) Laboratory for Theoretical Cosmology, Tomsk State University of \\ Control Systems and Radioelectronics, 634050 Tomsk, Russia (TUSUR)
}

\begin{abstract}
In this work we shall study the implications of a subclass of $E$-models cosmological attractors, namely of $a$-attractors, on hydrodynamically stable slowly rotating neutron stars. Specifically, we shall present the Jordan frame theory of the $a$-attractors, and by using a conformal transformation we shall derive the Einstein frame theory. We discuss the inflationary context of $a$-attractors in order to specify the allowed range of values for the free parameters of the model based on the latest cosmic-microwave-background-based Planck 2018 data. Accordingly, using the notation and physical units frequently used in theoretical astrophysics contexts, we shall derive the TolmanOppenheimer-Volkoff equations in the Einstein frame. Assuming a piecewise polytropic equation of state, the lowest density part of which shall be chosen to be the WFF1, or APR or the SLy EoS, we numerically solve the Tolman-Oppenheimer-Volkoff equations using a double shooting pythonbased "LSODA" numerical code. The resulting picture depends on the value of the parameter $a$ characterizing the $a$-attractors. As we show, for large values of $a$, which do not produce a viable inflationary era, the $M-R$ graphs are nearly identical to the general relativistic result, and these two are discriminated at large central densities values. Also, for large $a$-values, the WFF1 equation of state is excluded, due to the GW170817 constraints on the radius of an $M \sim 1.6 M_{\odot}$ neutron star, which must be larger than $R=10.68_{-0.04}^{+15} \mathrm{~km}$ and on the radius corresponding to the maximum mass which must be larger than $R=9.6_{-0.03}^{+0.14} \mathrm{~km}$. In addition, the small $a$ cases produce larger masses and radii compared to the general relativistic case and are compatible with the GW170817 constraints on the radii of neutron stars. A notable feature is that as the parameter $a$ decreases, the radii of the static hydrodynamically stable neutron stars increase. Our results indicate deep and not yet completely understood connections between non-minimal inflationary attractors and neutron stars phenomenology in scalar-tensor theory.
\end{abstract}

PACS numbers: 04.50.Kd, 95.36.+x, 98.80.-k, 98.80.Cq,11.25.-w

\section{Introduction}

After the exciting results of the LIGO-Virgo collaboration obtained in the last five years, neutron stars (NS) enjoy an elevated role in the scientific community, since many scientific theoretical frameworks can be tested by using neutron stars. For example nuclear theory models [1-11, high energy particle models [12 [15, modified gravity models [16 22] can be tested, but also NS are in the epicenter of also astrophysical models [23 30]. The two most important observations coming from the LIGO-Virgo collaboration are the GW170817 event 31] and the GW190814 event 32 , with the first indicating that the gravitational wave speed is nearly equal to the speed of light, and the latter indicating the possibility of either having an astrophysical black hole or a NS with mass belonging to the mass-gap region, although alternative scenarios can also be true 33. For an important stream of textbooks and reviews on NS we refer the reader to Refs. [34 38].

Modified gravity in its various forms [39 46], enjoys an elevated role among the various theoretical descriptions that can harbor larger NS masses compared to general relativity (GR). The most important and fundamental candidate modified gravity theory, namely $f(R)$ gravity, can yield large mass NSs [18, and solve the hyperon puzzle for a hyperon containing equation of state (EoS) [19. Apart from the applications in NSs, modified gravity seems as an inevitable description for cosmology, since dark energy cannot be consistently described solely by GR. The reason is simple, the dark energy EoS $\omega_{D E}$ according to the latest Planck cosmological constraints [47, is constrained to have values $\omega_{D E}=-1.018 \pm 0.031$. So it is a probability, not just a possibility, that dark energy is actually phantom dark energy. Phantom dark energy in the context of GR is described only by phantom scalars [48, which do not provide a physically acceptable description of nature. On the other hand, crossing the phantom divide line in the context of modified gravity is not an issue, since this can be achieved without resorting to phantom scalar fields, see the reviews 39 45 for details.

In view of the above, in this work we shall study static NSs in hydrodynamic equilibrium in the context of scalartensor gravity. Scalar-tensor studies of NSs already exist in the literature for various theories, see for example [49 62], but in this work we shall examine some $a$-attractor scalar models 63 99 which are unknown to the theoretical 
astrophysics community, but are quite popular in theoretical cosmology contexts. This is due to the fact that the cosmological attractors 63 99] have a main characteristic, they predict the same final form of the spectral index of the primordial scalar curvature perturbations and of the tensor-to-scalar ratio, for a wide class of distinct scalar potentials and non-minimal coupling. More importantly, the aforementioned indices are compatible in most cases with the latest Planck constraints on inflation 100. Our aim is to study in detail the Einstein frame counterpart implications on static NS in hydrodynamic equilibrium, of several classes of non-minimally coupled $a$-attractor models in the Jordan frame. We shall present both the inflationary theory, in order to better formulate the NS study, using the cosmologist's perspective, and then derive the Einstein frame Tolman-Oppenheimer-Volkoff (TOV) equations using Geometrized units and by adopting the usual notation of theoretical astrophysics contexts. With regard to the EoS, we shall use a piecewise polytropic EoS [101, 102, and in our study we shall extract from the numerical results the Jordan frame Arnowitt-Deser-Misner (ADM) mass [103, which for static NSs coincides [104] with the Komar mass [105. The numerical analysis will be based on a modified version of the freely available python 3 based numerical code [106], with the TOV equations changed in order to align the code with Ref. [49], and also the Jordan frame radii and masses of the NSs are calculated, not just the Einstein frame ones.

\section{I. a-ATTRACTORS INFLATION AND THEORETICAL FRAMEWORK}

Before we present the TOV equations in the Einstein frame, using the standard notation used in theoretical astrophysics context, let us first recall the theoretical framework of inflationary dynamics of $a$-attractor potentials, see Refs. 63 99 for details. Also in order to render the article self-contained, we shall recall to some limited extent the conformal transformation notation from the cosmologist perspective, see [107 110] for more details.

The Jordan frame action of non-minimally coupled scalar field in the presence of perfect matter fluids is,

$$
\mathcal{S}_{J}=\int d^{4} x\left[f(\phi) R-\frac{\omega(\phi)}{2} g^{\mu \nu} \partial_{\mu} \phi \partial_{\nu} \phi-U(\phi)\right]+S_{m}\left(g_{\mu \nu}, \psi_{m}\right),
$$

with $\psi_{m}$ being the Jordan frame perfect matter fluids present in the Jordan frame, which have pressure $P$ and energy density $\epsilon$. For this section we shall use natural units, however for the NS study we shall use Geometrized units, in order to comply with the NS literature and notation. In addition, the Jordan frame metric is $g_{\mu \nu}$ and the minimal coupling case in the action (1) corresponds to,

$$
f(\phi)=\frac{1}{16 \pi G}=\frac{M_{p}^{2}}{2}
$$

with,

$$
M_{p}=\frac{1}{\sqrt{8 \pi G}}
$$

which is the Jordan frame reduced Planck mass, while $G$ is Newton's gravitational constant in the Jordan frame. Upon performing the following conformal transformation,

$$
\tilde{g}_{\mu \nu}=\Omega^{2} g_{\mu \nu}
$$

the Einstein frame action is obtained, with the tilde denoting physical quantities in the Einstein frame. The minimal scalar field action in the Einstein frame is obtained by making the choice, [107, 108,

$$
\Omega^{2}=\frac{2}{M_{p}^{2}} f(\phi)
$$

hence upon performing the conformal transformation for all the quantities in the action (1) and for the choice (5), one obtains the Einstein frame action,

$$
\mathcal{S}_{E}=\int d^{4} x \sqrt{-\tilde{g}}\left[\frac{M_{p}^{2}}{2} \tilde{R}-\frac{\zeta(\phi)}{2} \tilde{g}^{\mu \nu} \tilde{\partial}_{\mu} \phi \tilde{\partial}_{\nu} \phi-V(\phi)\right]+S_{m}\left(\Omega^{-2} \tilde{g}_{\mu \nu}, \psi_{m}\right),
$$

with the Einstein frame scalar potential $V(\phi)$ being related to the Jordan frame one $U(\phi)$ as,

$$
V(\phi)=\frac{U(\phi)}{\Omega^{4}},
$$


and also $\zeta(\phi)$ is defined as follows,

$$
\zeta(\phi)=\frac{M_{p}^{2}}{2}\left(\frac{3\left(\frac{d f}{d \phi}\right)^{2}}{f^{2}}+\frac{2 \omega(\phi)}{f}\right) .
$$

We can make the scalar field kinetic term $\zeta(\phi)$ canonical, upon making the following transformation,

$$
\left(\frac{d \varphi}{d \phi}\right)=\sqrt{\zeta(\phi)}
$$

and in effect, the Einstein frame canonical scalar field action reads,

$$
\mathcal{S}_{E}=\int d^{4} x \sqrt{-\tilde{g}}\left[\frac{M_{p}^{2}}{2} \tilde{R}-\frac{1}{2} \tilde{g}^{\mu \nu} \tilde{\partial}_{\mu} \varphi \tilde{\partial}_{\nu} \varphi-V(\varphi)\right]+S_{m}\left(\Omega^{2} \tilde{g}_{\mu \nu}, \psi_{m}\right)
$$

where,

$$
V(\varphi)=\frac{U(\varphi)}{\Omega^{4}}=\frac{U(\varphi)}{4 M_{p}^{4} f^{2}} .
$$

It is important to note that in the Einstein frame, the matter fluids are not perfect, since the energy momentum tensor satisfies,

$$
\tilde{\partial}^{\mu} \tilde{T}_{\mu \nu}=-\frac{d}{d \varphi}[\ln \Omega] \tilde{T} \tilde{\partial}_{\nu} \phi
$$

where. Also, the pressure and the energy density are transformed from the Einstein to the Jordan frame as follows,

$$
\tilde{\varepsilon}=\Omega^{-4}(\varphi) \varepsilon, \quad \tilde{P}=\Omega^{-4}(\varphi) P .
$$

Let us now discuss how the $a$-attractor and the subclass of $E$-model attractor models are obtained. The $a$-attractor potentials can be obtained if we choose,

$$
\omega(\phi)=\frac{\left(\frac{d f}{d \phi}\right)^{2}}{4 \xi f},
$$

where $\xi$ is an arbitrary parameter of the model. By using the choice (14), the relation (9) becomes,

$$
\left(\frac{d \varphi}{d \phi}\right)=M_{p} \sqrt{\frac{3 a}{2}} \frac{\left(\frac{d f}{d \phi}\right)}{f},
$$

where we introduced the parameter $a$, defined as follows,

$$
a=1+\frac{1}{6 \xi} .
$$

Obviously, values of $a$ less than unity correspond to theories with negative $\xi$, while values of $a$ larger than unity correspond to positive $\xi$ values. In view of Eq. (9) and for the choice (15), we get,

$$
\varphi=M_{p} \sqrt{\frac{3 \alpha}{2}} \ln f
$$

and notice that the relation above holds true irrespective the choice of the arbitrary function $f$. Already the unique universality of the $a$-attractor potentials makes its presence apparent. From Eq. (17), we get,

$$
f=e^{\sqrt{\frac{2}{3 \alpha} \frac{\varphi}{M_{p}}}} .
$$

Now the $E$-models attractors and the $a$-attractors are obtained if the Jordan frame potential is generally chosen as,

$$
U(\phi)=V_{0} f^{2}\left(1-\frac{1}{f}\right)^{2 n},
$$


thus the Einstein frame potential reads,

$$
V(\varphi)=\mathcal{V}_{0}\left(1-\frac{1}{f}\right)^{2 n}=\tilde{V}_{0} M_{p}^{4}\left(1-\frac{1}{f}\right)^{2 n},
$$

where $\mathcal{V}_{0}=\tilde{V}_{0} M_{p}^{4}$ and $\tilde{V}_{0}=\frac{V_{0}}{4}$, thus $\tilde{V}_{0}$ is dimensionless in natural units and $\mathcal{V}_{0}$ has dimensions $[m]^{4}$. The class of potentials (19) for $f$ given in Eq. (18), is known as $E$-models potentials while for $n=2$ the much more popular $a$-attractor potentials are obtained. For all $a$ values both the $a$ and $E$-attractor potentials $(20)$ yield the same spectral index of the primordial scalar curvature perturbations $n_{s}$ and the same tensor-to-scalar ratio $r$ [65. We shall be interested in values of $a$ less than unity (the values can be extended to be of order $\mathcal{O}(10)$ and the theory can still be valid, see for example Figure 1 of Ref. [65], but we choose a safe value for $a$, namely $a=0.6$ ), and much larger than $\frac{8 N}{3}$, where $N$ is the $e$-foldings number which for successful inflation takes the values $N \sim 50-60$. Specifically for the NS study we shall choose $a=0.6$ and $a=10^{4}$, so let us discuss the inflationary dynamics of the two different limiting cases of $a$. As it was shown in 65, for $a$ being small or of the order of unity, the spectral index and the tensor-to-scalar ratio at leading order in $N$ are,

$$
n_{s} \simeq 1-\frac{2}{N}, r=\frac{12 a}{N^{2}} .
$$

Thus, regardless the choice of the function $f(\phi(\varphi))$, all the models 20 yield the same inflationary dynamics, and this justifies the terminology attractors. In this range, the dependence of the resulting phenomenology on $n$ is practically negligible. As we already mentioned, we shall focus on the case $n=1$, so we shall be interested in the $a$-attractors. The analysis of the NSs implications for general $n$ is similar, so we shall not discuss this case in this paper. The inflationary phenomenology implied by the observational indices of Eq. (21) is viable and compatible with the latest Planck data for a wide range of the parameter $a$, and certainly for $a$ of the order of unity (see Fig. 1 of Ref. 65. for details). Of course for $a=0.6$ the inflationary phenomenology is viable and the tensor-to-scalar ratio is smaller compared to the $R^{2}$ model. Also for $a \gg \frac{8 N}{3}$, the spectral index and the tensor-to-scalar ratio read [65],

$$
n_{s}=1-\frac{2}{N}, r=\frac{8}{N} .
$$

Note that the observational indices of inflation of Eq. [22 cannot be compatible with the Planck data [100] for $N \sim 50-60$, but we shall include this case in our NS study of the next section, since the potential 200 provides phenomenology which basically interpolates between the cases described by Eqs. (21) and (22). Interestingly enough, the limiting case $a \sim \mathcal{O}(1)$ which yields a viable inflationary phenomenology, results to NSs which deviate significantly from the GR case, while the large $a$ case results to NSs which mimic to some extent the GR behavior.

Before closing, let us demonstrate which values of the parameter $\tilde{V}_{0}$ defined below Eq. 20 can be compatible with the latest Planck constraints. According to the latest Planck data, the amplitude of the scalar fluctuations for canonical single field scalar models $\Delta_{s}^{2}$ defined as,

$$
\Delta_{s}^{2}=\frac{1}{24 \pi^{2}} \frac{V\left(\varphi_{f}\right)}{M_{p}^{4}} \frac{1}{\epsilon\left(\varphi_{f}\right)},
$$

is constrained to be [100,

$$
\Delta_{s}^{2}=2.2 \times 10^{-9} .
$$

In Eq. 23), $\varphi_{f}$ is the value of the scalar field at the end of inflation, and also $\epsilon$ is the first slow-roll index. This means that in our case,

$$
\mathcal{V}_{0}=\tilde{V}_{0} M_{p}^{4} \sim 9.6 \times 10^{-11} M_{p}^{4} .
$$

The above constraint shall be taken into account for the NSs study, because the parameter $\mathcal{V}_{0}$ cannot be arbitrary, and of course we shall transform the constraint 25) in Geometrized units and not natural units.

Before continuing, let us quote here a useful expression for the inflationary action in the Einstein frame,

$$
\mathcal{S}_{E}=\int d^{4} x \sqrt{-\tilde{g}}\left[\frac{M_{p}^{2}}{2} \tilde{R}-\frac{1}{2} \tilde{g}^{\mu \nu} \tilde{\partial}_{\mu} \varphi \tilde{\partial}_{\nu} \varphi-V(\varphi)\right]
$$

which can be rewritten as,

$$
\mathcal{S}_{E}=\int d^{4} x \sqrt{-\tilde{g}}\left[\frac{1}{16 \pi G} \tilde{R}-\frac{1}{2} \tilde{g}^{\mu \nu} \tilde{\partial}_{\mu} \varphi \tilde{\partial}_{\nu} \varphi-\frac{16 \pi G V(\varphi)}{16 \pi G}\right],
$$

where recall that $M_{p}^{2}=\frac{1}{8 \pi G}$. We shall make use of Eq. 27) for our next section NS study. 


\section{NEUTRON STARS IN THE EINSTEIN FRAME WITH $a$-ATTRACTORS SCALAR POTENTIAL}

Now we proceed to the study of NSs for $a$-attractor potentials we discussed in the previous section. We shall adopt though a different notation from the previous section in order to align the presentation with the theoretical astrophysics works, so we use the notation of Ref. [49] with the only difference being the use of tilde for the Einstein frame quantities. The general Jordan frame starting action of the non-minimally coupled scalar field in Geometrized units $(G=c=1)$ is 49 ,

$$
\mathcal{S}=\int d^{4} x \frac{\sqrt{-g}}{16 \pi}\left[f(\phi) R-\omega(\phi) g^{\mu \nu} \partial_{\mu} \phi \partial_{\nu} \phi-U(\phi)\right]+S_{m}\left(\psi_{m}, g_{\mu \nu}\right) .
$$

By performing the following conformal transformation,

$$
\tilde{g}_{\mu \nu}=A^{-2} g_{\mu \nu}
$$

and by choosing,

$$
A(\phi)=f^{-1 / 2}(\phi)
$$

we may obtain the Einstein frame action, which may be expressed in terms of a canonical scalar field $\varphi$ as follows,

$$
\mathcal{S}=\int d^{4} x \sqrt{-\tilde{g}}\left(\frac{\tilde{R}}{16 \pi}-\frac{1}{2} \tilde{g}_{\mu \nu} \partial^{\mu} \varphi \partial^{\nu} \varphi-\frac{V(\varphi)}{16 \pi}\right)+S_{m}\left(\psi_{m}, A^{2}(\varphi) g_{\mu \nu}\right),
$$

with the scalar field $\phi$ and the Einstein frame scalar field being related as follows,

$$
\frac{d \varphi}{d \phi}=\frac{1}{\sqrt{4 \pi}} \sqrt{\left(\frac{3}{4} \frac{1}{f^{2}}\left(\frac{d f}{d \phi}\right)^{2}+\frac{\omega(\phi)}{2 f}\right)}
$$

The $a$-attractors potentials can be obtained by choosing,

$$
\omega(\phi)=\frac{1}{4 \xi} \frac{1}{f}\left(\frac{d f}{d \phi}\right)^{2}
$$

and also for the Jordan frame potential,

$$
U(\phi)=\mathcal{U}_{0} f^{2}\left(1-\frac{1}{f}\right)^{2 n}
$$

By using Eq. (33), Eq. (32) yields,

$$
\frac{d \varphi}{d \phi}=\sqrt{\frac{3 a}{16 \pi}} \frac{1}{f}\left(\frac{d f}{d \phi}\right),
$$

where again $a$ is defined in Eq. (16). Thus, by integrating 35 we get,

$$
f=e^{\sqrt{\frac{16 \pi}{3 a}} \varphi}
$$

and holds true regardless the choice of the function $f$. The Jordan frame potential is obtained by using the following relation,

$$
V(\varphi)=\frac{U(\phi)}{f^{2}},
$$

hence by combining Eqs. (34) and (36), we obtain the final form of the Einstein frame potential which is,

$$
V(\varphi)=\mathcal{U}_{0}\left(1-e^{-\sqrt{\frac{16 \pi}{3 a}} \varphi}\right)^{2 n}
$$

which will be the final form of the $a$-attractors potential in the Einstein frame in Geometrized units. One can easily observe that by putting $M_{p}=1 / \sqrt{8 \pi G}$ in Eq. $\sqrt{18}$, relation $\sqrt{18}$ is identical with Eq. 36 , and the same applies for the potential. As a function of $\varphi$, the conforma factor $A(\phi)$ reads,

$$
A(\varphi)=e^{\alpha \varphi}
$$


where $\alpha$ stands for,

$$
\alpha=-\frac{1}{2} \sqrt{\frac{16 \pi}{3 a}}
$$

and in practise, $\alpha(\varphi)$ has the following definition in general,

$$
\alpha(\varphi)=\frac{d A(\varphi)}{d \varphi}
$$

hence in the case at hand,

$$
a(\varphi)=\alpha=-\frac{1}{2} \sqrt{\frac{16 \pi}{3 a}} .
$$

In the same way, the scalar potential in the Einstein frame is expressed in terms of the parameter $\alpha$ as follows,

$$
V(\varphi)=\mathcal{U}_{0}\left(1-e^{2 \alpha \varphi}\right)^{2 n}
$$

Now let us determine the values of $\mathcal{U}_{0}$ by making a direct correspondence to the inflationary theory of the previous
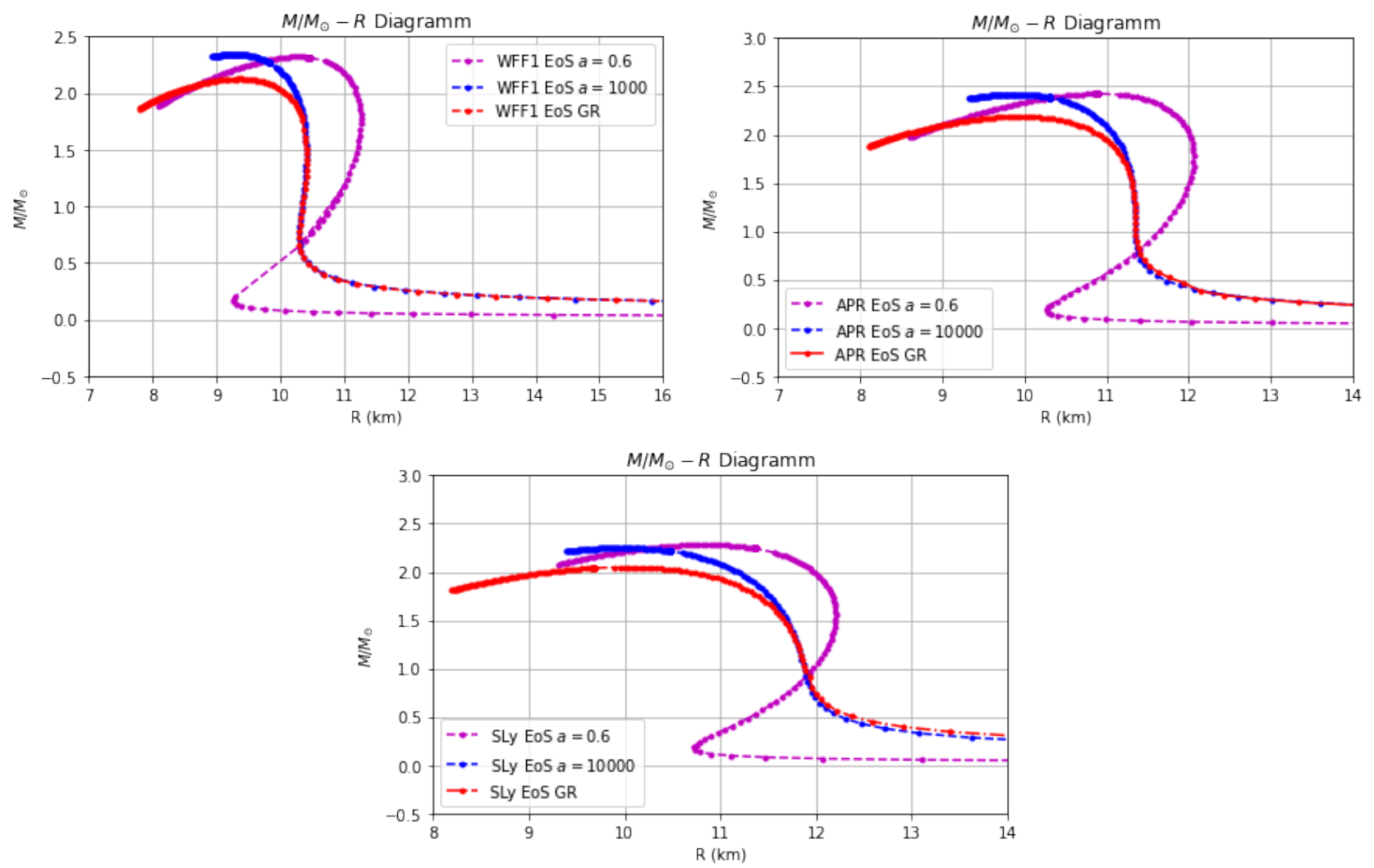

FIG. 1: $M-R$ graphs for the a-attractors model compared to the GR case, for the WFF1 EoS (upper left plot), the APR EoS (upper right plot) and the SLy EoS (bottom plot). The $y$-axis corresponds to $M / M_{\odot}$, where $M$ is the Jordan frame ADM mass of the neutron star, and the $x$-axis corresponds to the Jordan frame circumferential radius of the neutron star expressed in kilometers. In all the plots we took $\mathcal{U}_{0}=7.62094 \times 10^{-12}$, and the values of $a$ are displayed in each plot.

section. Using Eq. $\sqrt{27}$ and Eq. $\sqrt{25}$ a direct consequence is that $\mathcal{U}_{0}$ in Eq. $\sqrt{43}$ is $\mathcal{U}_{0}=16 \pi \mathcal{V}_{0}$ and since $M_{p}=1 / \sqrt{8 \pi}$ in Geometrized units, we get,

$$
\mathcal{U}_{0}=7.62094 \times 10^{-12},
$$

and this is the value for the scalar potential parameter $\mathcal{U}_{0}$ in Geometrized units. Now we proceed to the TOV equations for the framework at hand, and we shall consider a spherically symmetric and static metric of the form,

$$
d s^{2}=-e^{\nu(r)} d t^{2}+\frac{d r^{2}}{1-\frac{2 m}{r}}+r^{2}\left(d \theta^{2}+\sin ^{2} \theta d \phi^{2}\right)
$$


with the function $m(r)$ describing the gravitational mass of the neutron star of circumferential radius $r$. For the
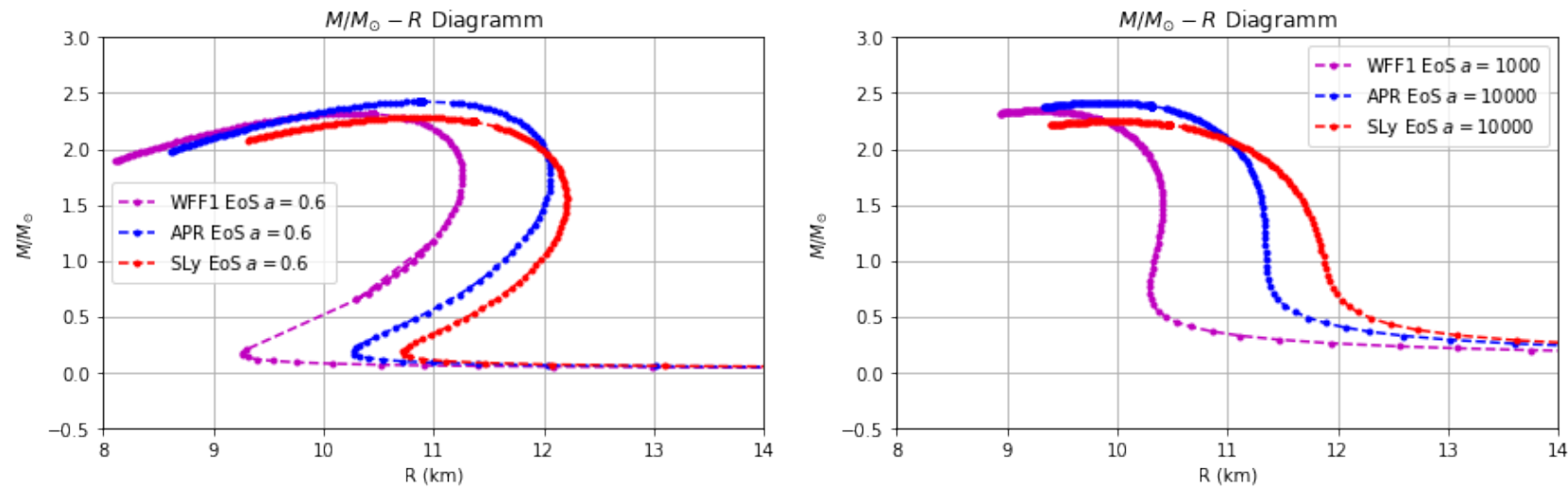

FIG. 2: Comparison of the $M-R$ graphs for the $a$-attractors model for each value of the parameter $a$ for all the EoSs. In the left plot we present the $M-R$ graphs for the WFF1, APR and SLy EoSs for $a=0.6$ and in the right plot for $a=1000$ for the WFF1 EoS, and for $a=10000$ for the SLy and APR EoSs.

metric 445, the TOV equations are [49,

$$
\begin{gathered}
\frac{d m}{d r}=4 \pi r^{2} A^{4}(\varphi) \epsilon+\frac{r}{2}(r-2 m) \omega^{2}+4 \pi r^{2} V(\varphi), \\
\frac{d \nu}{d r}=r \omega^{2}+\frac{2}{r(r-2 m)}\left[4 \pi A^{4}(\varphi) r^{3} P-4 \pi V(\varphi) r^{3}\right]+\frac{2 m}{r(r-2 m)}, \\
\frac{d \omega}{d r}=\frac{4 \pi r A^{4}(\varphi)}{r-2 m}(\alpha(\varphi)(\epsilon-3 P)+r \omega(\epsilon-P))-\frac{2 \omega(r-m)}{r(r-2 m)}+\frac{8 \pi \omega r^{2} V(\varphi)+r \frac{d V(\varphi)}{d \varphi}}{r-2 m}, \\
\frac{d P}{d r}=-(\epsilon+P)\left[\frac{1}{2} \frac{d \nu}{d r}+\alpha(\varphi) \omega\right]
\end{gathered}
$$

where we defined the function $\alpha(\varphi)$ in Eqs. 41) and (42) for the $a$-attractor model. Furthermore, there is an additional equation to be considered along with the other differential equations above, namely $\omega=\frac{d \varphi}{d r}$. Moreover, the pressure $P$ and energy density $\epsilon$ appearing in the TOV equations above, are basically the Jordan frame quantities, which are related to their Einstein frame counterparts as follows,

$$
\begin{aligned}
& \tilde{\epsilon}=A^{4} \epsilon, \\
& \tilde{P}=A^{4} P .
\end{aligned}
$$

So the purpose of this section is to numerically solve in a consistent way the TOV equations, and to this end we shall consider the following initial conditions,

$$
\begin{gathered}
P(0)=P_{c}, \\
m(0)=0, \\
\nu(0)=-\nu_{c} \\
\varphi(0)=\varphi_{c},
\end{gathered}
$$




$$
\omega(0)=0,
$$

and we shall be choosing three different types of EoSs for the neutron star nuclear matter. Specifically we shall consider piecewise polytropic EoSs [101, 102] (see also the text in [106]), in which the low-density part corresponds to the WFF1, APR or the SLy EoS. It is worth describing in brief the polytropic EoS in order to maintain the article self-contained. A piecewise polytropic EoS is constructed by a low-density part $\rho<\rho_{0}$, which is chosen to be a tabulated crust EoS, and by a high density part $\rho \gg \rho_{0}$. The density $\rho_{0}$ is obtained by matching the low and high density pieces. The piecewise polytropic EoS further consists of other two dividing high densities, $\rho_{1}=10^{14.7} \mathrm{~g} / \mathrm{cm}^{3}$ and $\rho_{2}=10^{15.0} \mathrm{~g} / \mathrm{cm}^{3}$, and for each of these densities, the intermediate densities and pressures $\rho_{i-1} \leq \rho \leq \rho_{i}$ satisfy,

$$
P=K_{i} \rho^{\Gamma_{i}},
$$

with the requirement of continuity in each of the crossing points of each piece, as follows,

$$
P\left(\rho_{i}\right)=K_{i} \rho^{\Gamma_{i}}=K_{i+1} \rho^{\Gamma_{i+1}} .
$$

Due to the continuity at each piece of the piecewise polytropic EoS, by using Eq. (58) one determines the parameters $K_{2}$ and $K_{3}$ for a given chosen values of the parameters $K_{1}, \Gamma_{1}, \Gamma_{2}, \Gamma_{3}$, or basically for a given initial pressure $p_{1}$ and for given parameters $\Gamma_{2}$, and $\Gamma_{3}$. In this work we shall consider three types of EoS, the a variational method EoS the WFF1 [11], a potential method EoS the SLy [112, and the APR EoS [113], so the parameters $p_{1}$ and the parameters $\Gamma_{2}$, and $\Gamma_{3}$ will be chosen according to the values determined by the above EoSs. Now let us quote the relation of the energy density as a function of the pressure for the piecewise polytropic EoS, which can be derived by simply integrating the first law of thermodynamics,

$$
d \frac{\epsilon}{\rho}=-P d \frac{1}{\rho}
$$

for a barotropic fluid, and by using the continuity of the energy density we get,

$$
\epsilon(\rho)=\left(1+\alpha_{i}\right) \rho+\frac{K_{i}}{\Gamma_{i}-1} \rho^{\Gamma_{i}},
$$

which holds true for $\Gamma_{i} \neq 1$, and also $\alpha_{i}$ is,

$$
\alpha_{i}=\frac{\epsilon\left(\rho_{i-1}\right)}{\rho_{i-1}}-1-\frac{K_{i}}{\Gamma_{i}-1} \rho_{i-1}^{\Gamma_{i}-1} .
$$

Before integrating the TOV equations, we need to clarify the mass of the neutron star issue, and we shall use the ADM mass. The numerical integration we shall perform on the TOV equations, shall yield the Einstein frame ADM mass, but we need to extract the Jordan frame ADM mass of the NS, since it is the Jordan frame mass which can be measured in observation via observing the Keplerian weak field orbits. Let us proceed to express the Jordan frame mass in terms of the Einstein frame mass and in terms of the scalar field. Let $h_{E}$ and $h_{J}$ be defined as follows in Geometrized units,

$$
\begin{aligned}
& \mathcal{K}_{E}=1-\frac{2 m}{r_{E}}, \\
& \mathcal{K}_{J}=1-\frac{2 m_{J}}{r_{J}} .
\end{aligned}
$$

The relation between $\mathcal{K}_{E}$ and $\mathcal{K}_{J}$ in the two frames is,

$$
\mathcal{K}_{J}=A^{-2} \mathcal{K}_{E},
$$

and furthermore the radii of the NS in the two frames satisfy,

$$
r_{J}=A r_{E},
$$

and the Jordan frame ADM mass is defined as follows,

$$
M_{J}=\lim _{r \rightarrow \infty} \frac{r_{J}}{2}\left(1-\mathcal{K}_{J}\right),
$$


while the Einstein frame ADM mass is defined as follows,

$$
M_{E}=\lim _{r \rightarrow \infty} \frac{r_{E}}{2}\left(1-\mathcal{K}_{E}\right) .
$$

If the asymptotic limit of Eq. 64 is taken, we have,

$$
\mathcal{K}_{J}\left(r_{E}\right)=\left(1+\alpha\left(\varphi\left(r_{E}\right)\right) \frac{d \varphi}{d r} r_{E}\right)^{2} \mathcal{K}_{E}\left(\varphi\left(r_{E}\right)\right)
$$

and hereafter $r_{E}$ will stand for the Einstein frame radius at large distances from the surface of the NS, near the numerical infinity but not exactly at the numerical infinity, and also $\frac{d \varphi}{d r}=\left.\frac{d \varphi}{d r}\right|_{r=r_{E}}$. By combining Eqs. $63-(68$ we finally get,

$$
M_{J}=A\left(\varphi\left(r_{E}\right)\right)\left(M_{E}-\frac{r_{E}^{2}}{2} \alpha\left(\varphi\left(r_{E}\right)\right) \frac{d \varphi}{d r}\left(2+\alpha\left(\varphi\left(r_{E}\right)\right) r_{E} \frac{d \varphi}{d r}\right)\left(1-\frac{2 M_{E}}{r_{E}}\right)\right)
$$

and recall that $\frac{d \varphi}{d r}=\left.\frac{d \varphi}{d r}\right|_{r=r_{E}}$. For our numerical analysis, after calculating the Einstein frame mass, we shall use the relation 69 in order to extract the Jordan frame mass, and also with $M$ we will denote the Jordan frame mass $M_{J}$, namely $M=M_{J}$.

Turning our focus to the neutron star radius, the Einstein frame one $R_{s}$ is determined by the condition $P\left(R_{s}\right)=0$, thus the Jordan frame radius of the NS, namely $R$, will be calculated by using the following formula,

$$
R=A\left(\varphi\left(R_{s}\right)\right) R_{s},
$$

and the result will be expressed in kilometers. Our aim in this section is to numerically solve the TOV equations for the interior and the exterior of the NS (where $\epsilon=P=0$ and the scalar potential is non-zero), and we shall extract the Jordan frame mass and radius of the NS and construct the corresponding $M-R$ graph. The numerical code we shall use is a modified version of the python 3 based code pyTOV-STT [106], using the "LSODA" numerical integrator, for which the Jordan frame mass and radius will be calculated. The numerical analysis will include a double shooting method in order to extract the optimal values of $\nu_{c}$ and $\varphi_{c}$ at the center of the NS, which provide a sufficient decay of the values of the Einstein frame scalar field and of the metric function $\nu(r)$ at the numerical infinity, which is chosen to be $r \sim 67.94378528694695 \mathrm{~km}$ in the Einstein frame. As we already mentioned, we shall use piecewise polytropic EoSs, with the parameters $p_{1}, \Gamma_{2}$, and $\Gamma_{3}$ corresponding to the WFF1 [111, the SLy [112, and the APR EoS [113. Finally, we shall study the cases for which $a=0.6$ and $a=10000$ for the APR and SLy, and also the cases $a=0.6$ and $a=1000$ for the WFF1.

The results of our numerical analysis can be seen in Figs. 1 and 2 , and now we shall critically examine the results of our analysis. In Fig. 1 we present the $M-R$ graphs for the $a$-attractors model compared to the GR case, for the WFF1 EoS (upper left plots), the APR EoS (upper right plots) and the SLy EoS (bottom plots). The $y$-axis corresponds to $M / M_{\odot}$, where $M$ is the Jordan frame ADM mass of the neutron star, and the $x$-axis corresponds to the Jordan frame circumferential radius of the neutron star $R$ expressed in kilometers. In all the plots we took $\mathcal{U}_{0}=7.62094 \times 10^{-12}$, and the values of $a$ are displayed in each plot. As it can be seen from all the plots, the large $a$ curves mimic significantly the corresponding GR curve, for a large number of central densities, and deviate from the GR curve from a certain point of high central density. Readily, the large $a$ case for the WFF1 EoS is excluded from being a viable NS configuration, since the GW170817 event constrains neutron stars with masses $M \sim 1.6 M_{\odot}$ area, to have radii in the range $R=10.68_{-0.04}^{+15} \mathrm{~km}[30$. Thus only for the WFF1 EoS, the large $a$ values are excluded, and it is remarkably interesting that for large $a$ values, the corresponding inflationary theory is not compatible with the Planck constraints on inflation. On the other hand, the small $a$ case for the WFF1 EoS is compatible with the aforementioned constraint of the GW170817 event. A common feature of all the $M-R$ curves for $a$ values near unity is that the stable NS configurations have larger radii, and specifically, as the values of $a$ decrease, the radii of the NSs increase. This can be seen from all the plots of Fig. 1 but also in Table I where we present the maximum masses for the $a$-attractor models and the correspondent radii. From Table [1] in conjunction with Fig. 1, we can also verify that the GW170817 constraint [30, which constrains the radii of the maximum mass static configurations to be larger than $R=9.6_{-0.03}^{+0.14} \mathrm{~km}$, is satisfied for all the cases studied, except for $a=1000$ for the WFF1 EoS which is excluded. In order to quantify the effect brought along by the different values of the parameter $a$, in Fig. 2 we present the same $M-R$ graphs corresponding to distinct values of the parameter $a$ for the three different EoSs we used. The left plot corresponds to $a=0.6$ while the right plot corresponds to $a=1000$ for the WFF1 EoS and $a=10^{4}$ for the APR and SLy EoS. From Fig. 2 one can see the effect of decreasing the value of $a$, which is the increase of the radius of static NSs in hydrodynamic equilibrium. 
TABLE I: Maximum Masses and the Corresponding Radii of Static NS for the a-attractors and for GR

\begin{tabular}{|r|r|r|r|}
\hline Model & APR EoS & SLy EoS & WFF1 EoS \\
\hline GR & $M_{\max }=2.18739372 M_{\odot}$ & $M_{\max }=2.04785291 M_{\odot}$ & $M_{\max }=2.12603999 M_{\odot}$ \\
\hline$a$-attractors $a=0.6$ & $M_{\max }=2.42368632 M_{\odot}$ & $M_{\max }=2.32152569 M_{\odot}$ & $M_{\max }=2.27222814 M_{\odot}$ \\
\hline$a$-attractors Radii $a=0.6$ & $R=10.89673795 \mathrm{~km}$ & $R=10.81663975 \mathrm{~km}$ & $R=9.8993922 \mathrm{~km}$ \\
\hline$a$-attractors large $a$ & $M_{\max }=2.41736711 M_{\odot}$ & $M_{\max }=2.24807309 M_{\odot}$ & $M_{\max }=2.34266656 M_{\odot}$ \\
\hline$a$-attractors Radii large $a$ & $R=10.35829055 \mathrm{~km}$ & $R=9.98770954 \mathrm{~km}$ & $R=9.28095863 \mathrm{~km}$ \\
\hline
\end{tabular}

Before closing we need to mention another class of $a$-attractor potentials which yield exactly the same theory in the Einstein frame, with the same conformal factor. The Jordan frame action in Geometrized units for these alternative $a$-attractors is,

$$
\mathcal{S}=\int d^{4} x \frac{\sqrt{-g}}{16 \pi}\left[f(\phi) R-\omega(\phi) g^{\mu \nu} \partial_{\mu} \phi \partial_{\nu} \phi-U_{J}(\phi)\right]+S_{m}\left(\psi_{m}, g_{\mu \nu}\right),
$$

with the potential $U(\phi)$ being equal to,

$$
U_{J}=\mathcal{U}_{0}(1-f(\phi))^{2}
$$

Choosing again the kinetic coupling function $\omega(\phi)$ as in Eq. [33), the function $f(\phi)$ as a function of the canonical Einstein frame scalar field is given again by Eq. (36), while the resulting Einstein frame potential is given again by the potential (43) for $n=1$. In addition, our numerical analysis indicated that by choosing a different number $n$ in the potential (43), does not change significantly the resulting NS phenomenology.

In view of our results, a strong question is whether the inflationary attractor behavior continues to NSs phenomenology too. This question is difficult to answer for the moment, because we need more data and analysis of several inflationary attractor potentials. However, one is certain, that a whole class of different arbitrary types of non-minimal couplings in the Jordan frame, with three different classes of potentials yield similar results.

\section{Concluding Remarks}

In this work we investigated the implications of a class of viable inflationary scalar potentials on NS. Specifically we were interested on $a$-attractor potentials which are known to provide not only a viable inflationary era, but also predict the same form for the spectral index of the primordial curvature perturbations and for the tensor-to-scalar ratio, regardless the functional form of the non-minimal coupling in the Jordan frame. The $a$-attractor potentials contain a parameter $a$, which when it takes values near unity or much smaller than unity, the resulting inflationary phenomenology is viable. On the contrary when the parameter $a$ takes large values, the inflationary phenomenology is not viable. After presenting the mechanism of obtaining the $a$-attractor potentials in the Einstein frame, starting from a class of Jordan frame models, we calculated the TOV equations for a spherically symmetric and static metric in the Einstein frame, having in mind that for the results we need to convert the Einstein frame quantities to their Jordan frame counterparts. With regard to the EoS, we used a piecewise polytropic EoS, with the low density part being the WFF1 or the APR or the SLy EoS. Accordingly, we numerically solved the TOV equations using a python 3 based numerical code, and we derived the $M-R$ graphs for the $a$-attractor theory. The resulting picture was quite interesting, since for all the studied cases, when small central densities and large $a$ vales are considered, the $M-R$ graphs of GR and of the $a$-attractor models coincide, however for larger central densities, the two graphs become clearly different. It is interesting to recall that the inflationary theory for large values of $a$ is not viable. Now, when small values of $a$ are considered, $a$-attractor $M-R$ diagrams deviated significantly from the corresponding GR graphs. Interestingly enough for small values of $a$, the inflationary theory is viable and compatible with the latest Planck observational constraints on inflation. Moreover, in all the cases, except for the WFF1 case for large $a$, the radii of the NSs for the $a$-attractor models satisfy both the GW170817 constraints derived in Ref. [30, namely that the radius of a $M \sim 1.6 M_{\odot}$ NS must be larger than $R=10.68_{-0.04}^{+15} \mathrm{~km}$, and the radii of the NSs corresponding to the maximum mass configurations, must be larger than $R=9.6_{-0.03}^{+0.14} \mathrm{~km}$. These constraints are violated for the WFF1 EoS, only for large $a$, since the theory mimics basically GR for those values of $a$. Before closing we need to mention that the $a$-attractor $M-R$ graphs seem to interpolate between the GR and the small $a$ a-attractors, for intermediate central densities however. Interestingly enough, the large $a$ potentials are essentially $\sim \varphi^{2}$ potentials. An important 
issue we did not address is whether the observed attractor behavior in the inflationary theories, continues to the level of NS, quantified differently of course. It seems that our preliminary results indicate deep and not yet completely understood connections between non-minimal inflationary attractors potentials and NSs phenomenology in scalartensor theory. In fact the inflationary attractor behavior might or might not be observed in NSs phenomenology, and in both cases the result will be interesting. In detail, if the attractor behavior is observed, this will be a direct correspondence between the inflationary and NS phenomenological theories. In the case that the attractor behavior is not observed, this will be a direct way of discriminating inflationary attractors at the NS level. For the moment we do not have enough data to address this important issue, and we hope to address this in a future work. Another issue that is worth discussing, is the Einstein-Gauss-Bonnet extension of the present theories [14, 119, which is string theory motivated. This study can be done for both the minimal and non-minimal couplings of the scalar field to the Ricci scalar, since there is no correspondence between the minimal and non-minimal couplings when the Gauss-Bonnet coupling is present. Thus only the Jordan frame theory can be studied. We hope to address some of these issues in a future work.

\section{Acknowledgments}

V.K. Oikonomou is indebted to N. Stergioulas and his MSc student Vaggelis Smyrniotis for the many hours spend on neutron star physics discussions and for sharing his professional knowledge on numerical integration of neutron stars in python. This work was supported by MINECO (Spain), project PID2019-104397GB-I00 and PHAROS COST Action (CA16214) (SDO).

[1] L. Tolos and L. Fabbietti, Prog. Part. Nucl. Phys. 112 (2020) 103770 doi:10.1016/j.ppnp.2020.103770 arXiv:2002.09223 [nucl-ex]].

[2] J. M. Lattimer, Ann. Rev. Nucl. Part. Sci. 62 (2012), 485-515 doi:10.1146/annurev-nucl-102711-095018 arXiv:1305.3510 [nucl-th]].

[3] A. W. Steiner and S. Gandolfi, Phys. Rev. Lett. 108 (2012), 081102 doi:10.1103/PhysRevLett.108.081102 arXiv:1110.4142 [nucl-th]].

[4] C. J. Horowitz, M. A. Perez-Garcia, D. K. Berry and J. Piekarewicz, Phys. Rev. C 72 (2005), 035801 doi:10.1103/PhysRevC.72.035801 [arXiv:nucl-th/0508044 [nucl-th]].

[5] G. Watanabe, K. Iida and K. Sato, Nucl. Phys. A 676 (2000), 455-473 [erratum: Nucl. Phys. A 726 (2003), 357-365] doi:10.1016/S0375-9474(00)00197-4 arXiv:astro-ph/0001273 [astro-ph]].

[6] H. Shen, H. Toki, K. Oyamatsu and K. Sumiyoshi, Nucl. Phys. A 637 (1998), 435-450 doi:10.1016/S0375-9474(98)00236-X arXiv:nucl-th/9805035 [nucl-th]].

[7] J. Xu, L. W. Chen, B. A. Li and H. R. Ma, Astrophys. J. 697 (2009), 1549-1568 doi:10.1088/0004-637X/697/2/1549 arXiv:0901.2309 [astro-ph.SR]].

[8] K. Hebeler, J. M. Lattimer, C. J. Pethick and A. Schwenk, Astrophys. J. 773 (2013), 11 doi:10.1088/0004-637X/773/1/11 arXiv:1303.4662 [astro-ph.SR]].

[9] J. de Jesús Mendoza-Temis, M. R. Wu, G. Martínez-Pinedo, K. Langanke, A. Bauswein and H. T. Janka, Phys. Rev. C 92 (2015) no.5, 055805 doi:10.1103/PhysRevC.92.055805 arXiv:1409.6135 [astro-ph.HE]].

[10] W. C. G. Ho, K. G. Elshamouty, C. O. Heinke and A. Y. Potekhin, Phys. Rev. C 91 (2015) no.1, 015806 doi:10.1103/PhysRevC.91.015806 arXiv:1412.7759 [astro-ph.HE]].

[11] A. Kanakis-Pegios, P. S. Koliogiannis and C. C. Moustakidis, arXiv:2012.09580 [astro-ph.HE]].

[12] M. Buschmann, R. T. Co, C. Dessert and B. R. Safdi, Phys. Rev. Lett. 126 (2021) no.2, 021102 doi:10.1103/PhysRevLett.126.021102 arXiv:1910.04164 [hep-ph]].

[13] B. R. Safdi, Z. Sun and A. Y. Chen, Phys. Rev. D 99 (2019) no.12, 123021 doi:10.1103/PhysRevD.99.123021 arXiv:1811.01020 [astro-ph.CO]].

[14] A. Hook, Y. Kahn, B. R. Safdi and Z. Sun, Phys. Rev. Lett. 121 (2018) no.24, 241102 doi:10.1103/PhysRevLett.121.241102 arXiv:1804.03145 [hep-ph]].

[15] T. D. P. Edwards, B. J. Kavanagh, L. Visinelli and C. Weniger, arXiv:2011.05378 [hep-ph]].

[16] A. V. Astashenok, S. Capozziello, S. D. Odintsov and V. K. Oikonomou, Phys. Lett. B 811 (2020), 135910 doi:10.1016/j.physletb.2020.135910 arXiv:2008.10884 [gr-qc]].

[17] S. Capozziello, M. De Laurentis, R. Farinelli and S. D. Odintsov, Phys. Rev. D 93 (2016) no.2, 023501 doi:10.1103/PhysRevD.93.023501 arXiv:1509.04163 [gr-qc]].

[18] A. V. Astashenok, S. Capozziello and S. D. Odintsov, JCAP 01 (2015), 001 doi:10.1088/1475-7516/2015/01/001 arXiv:1408.3856 [gr-qc]].

[19] A. V. Astashenok, S. Capozziello and S. D. Odintsov, Phys. Rev. D 89 (2014) no.10, 103509 doi:10.1103/PhysRevD.89.103509 arXiv:1401.4546 [gr-qc]]. 
[20] A. V. Astashenok, S. Capozziello and S. D. Odintsov, JCAP 12 (2013), 040 doi:10.1088/1475-7516/2013/12/040 arXiv:1309.1978 [gr-qc]].

[21] A. S. Arapoglu, C. Deliduman and K. Y. Eksi, JCAP 07 (2011), 020 doi:10.1088/1475-7516/2011/07/020 arXiv:1003.3179 $[\mathrm{gr}-\mathrm{qc}]]$.

[22] A. V. Astashenok and S. D. Odintsov, Mon. Not. Roy. Astron. Soc. 498 (2020) no.3, 3616 doi:10.1093/mnras/staa2630 arXiv:2008.11271 [gr-qc]].

[23] A. Sedrakian, Phys. Rev. D 93 (2016) no.6, 065044 doi:10.1103/PhysRevD.93.065044 arXiv:1512.07828 [astro-ph.HE]].

[24] S. Khadkikar, A. R. Raduta, M. Oertel and A. Sedrakian, arXiv:2102.00988 [astro-ph.HE].

[25] D. M. Sedrakian, M. V. Hayrapetyan and M. K. Shahabasyan, Astrophysics 49 (2006), 194-200 doi:10.1007/s10511-0060020-4

[26] A. Sedrakian, Phys. Rev. D 99 (2019) no.4, 043011 doi:10.1103/PhysRevD.99.043011 arXiv:1810.00190 [astro-ph.HE]].

[27] A. Bauswein, G. Guo, J. H. Lien, Y. H. Lin and M. R. Wu, arXiv:2012.11908 [astro-ph.HE]].

[28] S. Vretinaris, N. Stergioulas and A. Bauswein, Phys. Rev. D 101 (2020) no.8, 084039 doi:10.1103/PhysRevD.101.084039 arXiv:1910.10856 [gr-qc]].

[29] A. Bauswein, S. Blacker, V. Vijayan, N. Stergioulas, K. Chatziioannou, J. A. Clark, N. U. F. Bastian, D. B. Blaschke, M. Cierniak and T. Fischer, Phys. Rev. Lett. 125 (2020) no.14, 141103 doi:10.1103/PhysRevLett.125.141103 arXiv:2004.00846 [astro-ph.HE]].

[30] A. Bauswein, O. Just, H. T. Janka and N. Stergioulas, Astrophys. J. Lett. 850 (2017) no.2, L34 doi:10.3847/20418213/aa9994 arXiv:1710.06843 [astro-ph.HE]].

[31] B. P. Abbott et al. [LIGO Scientific and Virgo], Phys. Rev. Lett. 119 (2017) no.16, 161101 doi:10.1103/PhysRevLett.119.161101 [arXiv:1710.05832 [gr-qc]].

[32] R. Abbott et al. [LIGO Scientific and Virgo], Astrophys. J. Lett. 896 (2020) no.2, L44 doi:10.3847/2041-8213/ab960f arXiv:2006.12611 [astro-ph.HE]].

[33] B. Biswas, R. Nandi, P. Char, S. Bose and N. Stergioulas, arXiv:2010.02090 [astro-ph.HE]].

[34] P. Haensel, A. Y. Potekhin and D. G. Yakovlev, Astrophys. Space Sci. Libr. 326 (2007), pp.1-619 doi:10.1007/978-0-38747301-7

[35] J. L. Friedman and N. Stergioulas, doi:10.1017/CBO9780511977596

[36] G. Baym, T. Hatsuda, T. Kojo, P. D. Powell, Y. Song and T. Takatsuka, Rept. Prog. Phys. 81 (2018) no.5, 056902 doi:10.1088/1361-6633/aaae14 arXiv:1707.04966 [astro-ph.HE]].

[37] J. M. Lattimer and M. Prakash, Science 304 (2004), 536-542 doi:10.1126/science.1090720 arXiv:astro-ph/0405262 [astro$\mathrm{ph}]$.

[38] G. J. Olmo, D. Rubiera-Garcia and A. Wojnar, Phys. Rept. 876 (2020), 1-75 doi:10.1016/j.physrep.2020.07.001 arXiv:1912.05202 [gr-qc]].

[39] S. Nojiri, S. D. Odintsov and V. K. Oikonomou, Phys. Rept. 692 (2017) 1 doi:10.1016/j.physrep.2017.06.001 arXiv:1705.11098 [gr-qc]].

[40] S. Capozziello and M. De Laurentis, Phys. Rept. 509 (2011) 167 doi:10.1016/j.physrep.2011.09.003 arXiv:1108.6266 [gr-qc]].

[41] V. Faraoni and S. Capozziello, Fundam. Theor. Phys. 170 (2010). doi:10.1007/978-94-007-0165-6

[42] S. Nojiri and S. D. Odintsov, eConf C 0602061 (2006) 06 [Int. J. Geom. Meth. Mod. Phys. 4 (2007) 115] doi:10.1142/S0219887807001928 hep-th/0601213.

[43] S. Nojiri and S. D. Odintsov, Phys. Rept. 505 (2011) 59 doi:10.1016/j.physrep.2011.04.001 arXiv:1011.0544 [gr-qc]].

[44] A. de la Cruz-Dombriz and D. Saez-Gomez, Entropy 14 (2012) 1717 doi:10.3390/e14091717 arXiv:1207.2663 [gr-qc]].

[45] G. J. Olmo, Int. J. Mod. Phys. D 20 (2011) 413 doi:10.1142/S0218271811018925 [arXiv:1101.3864 [gr-qc]].

[46] Konstantinos Dimopoulos, Introduction to Cosmic Inflation and Dark Energy, (2021) CRC Press

[47] N. Aghanim et al. [Planck], Astron. Astrophys. 641 (2020), A6 doi:10.1051/0004-6361/201833910 arXiv:1807.06209 [astro-ph.CO]].

[48] R. R. Caldwell, M. Kamionkowski and N. N. Weinberg, Phys. Rev. Lett. 91 (2003), 071301 doi:10.1103/PhysRevLett.91.071301 [arXiv:astro-ph/0302506 [astro-ph]].

[49] P. Pani and E. Berti, Phys. Rev. D 90 (2014) no.2, 024025 doi:10.1103/PhysRevD.90.024025 [arXiv:1405.4547 [gr-qc]].

[50] K. V. Staykov, D. D. Doneva, S. S. Yazadjiev and K. D. Kokkotas, JCAP 10 (2014), 006 doi:10.1088/14757516/2014/10/006 arXiv:1407.2180 [gr-qc]].

[51] M. Horbatsch, H. O. Silva, D. Gerosa, P. Pani, E. Berti, L. Gualtieri and U. Sperhake, Class. Quant. Grav. 32 (2015) no.20, 204001 doi:10.1088/0264-9381/32/20/204001 arXiv:1505.07462 [gr-qc]].

[52] H. O. Silva, C. F. B. Macedo, E. Berti and L. C. B. Crispino, Class. Quant. Grav. 32 (2015), 145008 doi:10.1088/02649381/32/14/145008 arXiv:1411.6286 [gr-qc]].

[53] D. D. Doneva, S. S. Yazadjiev, N. Stergioulas and K. D. Kokkotas, Phys. Rev. D 88 (2013) no.8, 084060 doi:10.1103/PhysRevD.88.084060 arXiv:1309.0605 [gr-qc]].

[54] R. Xu, Y. Gao and L. Shao, Phys. Rev. D 102 (2020) no.6, 064057 doi:10.1103/PhysRevD.102.064057 arXiv:2007.10080 $[\mathrm{gr}-\mathrm{qc}]]$.

[55] M. Salgado, D. Sudarsky and U. Nucamendi, Phys. Rev. D 58 (1998), 124003 doi:10.1103/PhysRevD.58.124003 arXiv:grqc/9806070 [gr-qc]].

[56] M. Shibata, K. Taniguchi, H. Okawa and A. Buonanno, Phys. Rev. D 89 (2014) no.8, 084005 doi:10.1103/PhysRevD.89.084005 arXiv:1310.0627 [gr-qc]].

[57] A. Savaş Arapoğlu, K. Yavuz Ekşi and A. Emrah Yükselci, Phys. Rev. D 99 (2019) no.6, 064055 
doi:10.1103/PhysRevD.99.064055 arXiv:1903.00391 [gr-qc]].

[58] F. M. Ramazanoğlu and F. Pretorius, Phys. Rev. D 93 (2016) no.6, 064005 doi:10.1103/PhysRevD.93.064005 arXiv:1601.07475[gr-qc]].

[59] Z. Altaha Motahar, J. L. Blázquez-Salcedo, D. D. Doneva, J. Kunz and S. S. Yazadjiev, Phys. Rev. D 99 (2019) no.10, 104006 doi:10.1103/PhysRevD.99.104006 arXiv:1902.01277 [gr-qc]].

[60] X. Y. Chew, V. Dzhunushaliev, V. Folomeev, B. Kleihaus and J. Kunz, Phys. Rev. D 100 (2019) no.4, 044019 doi:10.1103/PhysRevD.100.044019 arXiv:1906.08742 [gr-qc]].

[61] J. L. Blázquez-Salcedo, F. Scen Khoo and J. Kunz, EPL 130 (2020) no.5, 50002 doi:10.1209/0295-5075/130/50002 arXiv:2001.09117 [gr-qc]].

[62] Z. Altaha Motahar, J. L. Blázquez-Salcedo, B. Kleihaus and J. Kunz, Phys. Rev. D 96 (2017) no.6, 064046 doi:10.1103/PhysRevD.96.064046 arXiv:1707.05280 [gr-qc]].

[63] R. Kallosh and A. Linde, JCAP 1307 (2013) 002 arXiv:1306.5220 [hep-th]].

[64] S. Ferrara, R. Kallosh, A. Linde and M. Porrati, Phys. Rev. D 88 (2013) no.8, 085038 arXiv:1307.7696 [hep-th]].

[65] R. Kallosh, A. Linde and D. Roest, JHEP 1311 (2013) 198 arXiv:1311.0472 [hep-th]].

[66] A. Linde, JCAP 05 (2015), 003 doi:10.1088/1475-7516/2015/05/003 arXiv:1504.00663 [hep-th]].

[67] S. Cecotti and R. Kallosh, JHEP 1405 (2014) 114 arXiv:1403.2932 [hep-th]].

[68] J. J. M. Carrasco, R. Kallosh and A. Linde, JHEP 1510 (2015) 147 arXiv:1506.01708 [hep-th]].

[69] J. J. M. Carrasco, R. Kallosh, A. Linde and D. Roest, Phys. Rev. D 92 (2015) no.4, 041301 doi:10.1103/PhysRevD.92.041301 arXiv:1504.05557 [hep-th]].

[70] D. Roest and M. Scalisi, Phys. Rev. D 92 (2015) 043525 doi:10.1103/PhysRevD.92.043525 arXiv:1503.07909 [hep-th]].

[71] R. Kallosh, A. Linde and D. Roest, JHEP 1408 (2014) 052 doi:10.1007/JHEP08(2014)052 arXiv:1405.3646 [hep-th]].

[72] J. Ellis, D. V. Nanopoulos and K. A. Olive, JCAP 1310 (2013) 009 [arXiv:1307.3537 [hep-th]].

[73] Y. F. Cai, J. O. Gong and S. Pi, Phys. Lett. B 738 (2014) 20 doi:10.1016/j.physletb.2014.09.009 arXiv:1404.2560 [hep-th]].

[74] Z. Yi and Y. Gong, arXiv:1608.05922 [gr-qc].

[75] Y. Akrami, R. Kallosh, A. Linde and V. Vardanyan, JCAP 06 (2018), 041 doi:10.1088/1475-7516/2018/06/041 arXiv:1712.09693 [hep-th]].

[76] S. Qummer, A. Jawad and M. Younas, Int. J. Mod. Phys. D 29 (2020) no.16, 2050117 doi:10.1142/S0218271820501175

[77] Q. Fei, Z. Yi and Y. Yang, Universe 6 (2020) no.11, 213 doi:10.3390/universe6110213 arXiv:2009.14819 [gr-qc]].

[78] A. D. Kanfon, F. Mavoa and S. M. J. Houndjo, Astrophys. Space Sci. 365 (2020) no.6, 97 doi:10.1007/s10509-020-03813-6

[79] I. Antoniadis, A. Karam, A. Lykkas, T. Pappas and K. Tamvakis, PoS CORFU2019 (2020), 073 doi:10.22323/1.376.0073 arXiv:1912.12757[gr-qc]].

[80] C. García-García, P. Ruíz-Lapuente, D. Alonso and M. Zumalacárregui, JCAP 07 (2019), 025 doi:10.1088/14757516/2019/07/025 arXiv:1905.03753 [astro-ph.CO]].

[81] F. X. Linares Cedeño, A. Montiel, J. C. Hidalgo and G. Germán, JCAP 08 (2019), 002 doi:10.1088/1475-7516/2019/08/002 arXiv:1905.00834 [gr-qc]].

[82] S. Karamitsos, JCAP 09 (2019), 022 doi:10.1088/1475-7516/2019/09/022 arXiv:1903.03707 [hep-th]].

[83] D. D. Canko, I. D. Gialamas and G. P. Kodaxis, Eur. Phys. J. C 80 (2020) no.5, 458 doi:10.1140/epjc/s10052-020-8025-4 arXiv:1901.06296 [hep-th]].

[84] T. Miranda, C. Escamilla-Rivera, O. F. Piattella and J. C. Fabris, JCAP 05 (2019), 028 doi:10.1088/14757516/2019/05/028 arXiv:1812.01287 [gr-qc]].

[85] A. Karam, T. Pappas and K. Tamvakis, JCAP 02 (2019), 006 doi:10.1088/1475-7516/2019/02/006 arXiv:1810.12884 $[\mathrm{gr}-\mathrm{qc}]]$.

[86] K. Nozari and N. Rashidi, Astrophys. J. 863 (2018) no.2, 133 doi:10.3847/1538-4357/aad18e arXiv:1808.05363 [astroph.CO]].

[87] C. García-García, E. V. Linder, P. Ruíz-Lapuente and M. Zumalacárregui, JCAP 08 (2018), 022 doi:10.1088/14757516/2018/08/022 arXiv:1803.00661 [astro-ph.CO]].

[88] N. Rashidi and K. Nozari, Int. J. Mod. Phys. D 27 (2018) no.07, 1850076 doi:10.1142/S0218271818500761 arXiv:1802.09185 [astro-ph.CO]].

[89] Q. Gao, Y. Gong and Q. Fei, JCAP 05 (2018), 005 doi:10.1088/1475-7516/2018/05/005 arXiv:1801.09208 [gr-qc]].

[90] K. Dimopoulos, L. Donaldson Wood and C. Owen, Phys. Rev. D 97 (2018) no.6, 063525 doi:10.1103/PhysRevD.97.063525 arXiv:1712.01760 [astro-ph.CO]].

[91] T. Miranda, J. C. Fabris and O. F. Piattella, JCAP 09 (2017), 041 doi:10.1088/1475-7516/2017/09/041 arXiv:1707.06457 $[\mathrm{gr}-\mathrm{qc}]]$.

[92] A. Karam, T. Pappas and K. Tamvakis, Phys. Rev. D 96 (2017) no.6, 064036 doi:10.1103/PhysRevD.96.064036 arXiv:1707.00984 [gr-qc]].

[93] K. Nozari and N. Rashidi, Phys. Rev. D 95 (2017) no.12, 123518 doi:10.1103/PhysRevD.95.123518 arXiv:1705.02617 [astro-ph.CO]].

[94] Q. Gao and Y. Gong, Eur. Phys. J. Plus 133 (2018) no.11, 491 doi:10.1140/epjp/i2018-12324-3 arXiv:1703.02220 [gr-qc]].

[95] C. Q. Geng, C. C. Lee and Y. P. Wu, Eur. Phys. J. C 77 (2017) no.3, 162 doi:10.1140/epjc/s10052-017-4720-1 arXiv:1512.04019 [astro-ph.CO]].

[96] S. D. Odintsov and V. K. Oikonomou, Phys. Lett. B 807 (2020), 135576 doi:10.1016/j.physletb.2020.135576 arXiv:2005.12804 [gr-qc]].

[97] S. D. Odintsov and V. K. Oikonomou, Phys. Rev. D 94 (2016) no.12, 124026 doi:10.1103/PhysRevD.94.124026 arXiv:1612.01126 [gr-qc]]. 
[98] S. D. Odintsov and V. K. Oikonomou, Class. Quant. Grav. 34 (2017) no.10, 105009 doi:10.1088/1361-6382/aa69a8 arXiv:1611.00738 [gr-qc]].

[99] L. Järv, A. Karam, A. Kozak, A. Lykkas, A. Racioppi and M. Saal, Phys. Rev. D 102 (2020) no.4, 044029 doi:10.1103/PhysRevD.102.044029 arXiv:2005.14571 [gr-qc]].

[100] Y. Akrami et al. [Planck], Astron. Astrophys. 641 (2020), A10 doi:10.1051/0004-6361/201833887 arXiv:1807.06211 [astroph.CO]].

[101] J. S. Read, B. D. Lackey, B. J. Owen and J. L. Friedman, Phys. Rev. D 79 (2009), 124032

[102] J. S. Read, C. Markakis, M. Shibata, K. Uryu, J. D. E. Creighton and J. L. Friedman, Phys. Rev. D 79 (2009), 124033

[103] R. Arnowitt, S. Deser and C. W. Misner, Phys. Rev. 118 (1960), 1100-1104 doi:10.1103/PhysRev.118.1100

[104] M. Shibata and K. Kawaguchi, Phys. Rev. D 87 (2013) no.10, 104031 doi:10.1103/PhysRevD.87.104031

[105] A. Komar, Phys. Rev. 113 (1959), 934-936 doi:10.1103/PhysRev.113.934

[106] Nikolaos Stergioulas, https://github.com/niksterg

[107] D. I. Kaiser, Phys. Rev. D 52 (1995), 4295-4306 doi:10.1103/PhysRevD.52.4295 arXiv:astro-ph/9408044 [astro-ph]].

[108] Valerio Faraoni, Cosmology in Scalar-Tensor Gravity, Springer 2004

[109] V. Faraoni, Galaxies 1 (2013) no.2, 96-106

[110] M. Buck, M. Fairbairn and M. Sakellariadou, Phys. Rev. D 82 (2010), 043509

[111] R. B. Wiringa, V. Fiks and A. Fabrocini, Phys. Rev. C 38 (1988), 1010-1037 doi:10.1103/PhysRevC.38.1010

[112] F. Douchin and P. Haensel, Astron. Astrophys. 380 (2001), 151 doi:10.1051/0004-6361:20011402 arXiv:astro-ph/0111092 [astro-ph]].

[113] A. Akmal, V. R. Pandharipande and D. G. Ravenhall, Phys. Rev. C 58 (1998), 1804-1828 doi:10.1103/PhysRevC.58.1804 arXiv:nucl-th/9804027 [nucl-th]].

[114] J. c. Hwang and H. Noh, Phys. Rev. D 71 (2005) 063536 doi:10.1103/PhysRevD.71.063536 gr-qc/0412126.

[115] P. Kanti, R. Gannouji and N. Dadhich, Phys. Rev. D 92 (2015) no.4, 041302 doi:10.1103/PhysRevD.92.041302 arXiv:1503.01579 [hep-th]].

[116] M. De Laurentis, M. Paolella and S. Capozziello, Phys. Rev. D 91 (2015) no.8, 083531 doi:10.1103/PhysRevD.91.083531 arXiv:1503.04659 [gr-qc]].

[117] Z. Yi and Y. Gong, Universe 5 (2019) no.9, 200 doi:10.3390/universe5090200 arXiv:1811.01625 [gr-qc]].

[118] B. Kleihaus, J. Kunz and P. Kanti, arXiv:1910.02121 [gr-qc].

[119] A. Bakopoulos, P. Kanti and N. Pappas, Phys. Rev. D 101 (2020) no.4, 044026 doi:10.1103/PhysRevD.101.044026 arXiv:1910.14637 [hep-th]]. 